\title{
Model-Based Reliability Analysis
}

The submitted manascript hes been authored by a contractor of the United States Government under contract. Accordingly the United States Government retains a non-exalusive, royalty-free license to publish or reproduce the published form of this contribution, or allow others to do 80, for United Stater Government pasposes.

Rene L. Bierbaum, Sandia National Laboratories, Livermore, CA Thomas D. Brown, Sandia National Laboratories, Albuquerque, NM Thomas J. Kerschen, Sandia National Laboratories, Albuquerque, NM

Key Words: CAD/CAE/CAM/CIM, Design margin, Electrical modeling, Life prediction, Modeling, SPICE, Weapon System

\section{SUMMARY \& CONCLUSIONS}

Modeling, in conjunction with testing, is a rich source of insight. Model parameters are easily controlled and monitoring can be done unobtrusively. The ability to inject faults without otherwise affecting performance is particularly critical. Many iterations can be done quickly with a model while varying parameters and conditions based on a small number of validation tests.

The objective of Model-Based Reliability Analysis (MBRA) is to identify ways to capitalize on the insights gained from modeling to make both qualitative and quantitative statements about product reliability. MBRA will be developed and exercised in the realm of weapon system development and maintenance, where the challenges of severe environmental requirements, limited production quantities, and use of one-shot devices can make testing prohibitively expensive. However, the general principles will also be applicable to other product types.

There are many anticipated benefits from MBRA, especially in the context of weapon systems:

- Development time and required test assets will be reduced. In addition, there will be fewer design iterations necessary. Fewer modifications in the production processes will be needed, leading to a more homogeneous product. Furthermore, MBRA can be used to evaluate the impact of production and part changes if they do become necessary.

- Typically it has been challenging in the past to determine the generalized impact of an observed anomaly (i.e., how the anomalous behavior may manifest itself under different but still valid - conditions). Often specific conditions cannot be varied in a controlled fashion in a testing situation. Use of a modeling framework permits one to inject hypothesized behaviors under different conditions and observe the consequences. This enables the effective leveraging of (often limited) test results that provide the initial foundation for model development.

\section{INTRODUCTION}

Recent improvements in physical response modeling capabilities coupled with declining resources for development and qualification of new product drive us to examine new means by which non-traditional data and knowledge obtained from modeling can be used to make inferences about reliability. The purpose of the Model-Based Reliability Analysis (MBRA) effort is to identify the areas where modeling can supplement our current reliability assessment 


\section{DISCLAIMER}

This report was prepared as an account of work sponsored by an agency of the United States Government. Neither the United States Government nor any agency thereof, nor any of their employees, make any warranty, express or implied, or assumes any legal liability or responsibility for the accuracy, completeness, or usefulness of any information, apparatus, product, or process disclosed, or represents that its use would not infringe privately owned rights. Reference herein to any specific commercial product, process, or service by trade name, trademark, manufacturer, or otherwise does not necessarily constitute or imply its endorsement, recommendation, or favoring by the United States Government or any agency thereof. The views and opinions of authors expressed herein do not necessarily state or reflect those of the United States Government or any agency thereof. 


\section{DISCLAIMER}

Portions of this document may be illegible in electronic image products. Images are produced from the best available original document. 
methodology, which is based primarily on the use of product test data obtained at various levels of assembly and under different environmental conditions.

A framework for using modeling in this context will first be described. Then the specific application of two elements of this framework to a weapon component will be described. The insights gained, as well as the limitations and caveats of the MBRA approach, will be discussed. Finally, the next steps in the MBRA project will be delineated.

\section{BACKGROUND}

The role of modeling in the reliability analysis process at Sandia National Laboratories is driven by the nature of the product being analyzed, the specific way in which Sandia defines reliability, the product test program, and the current assessment approach. The products are weapon systems that spend much of their multidecade lives in dormant storage. Although some of the subassemblies can be operated repeatedly, the weapon system is essentially a one-shot device. Further complicating the reliability analysis is that, due to treaty limitations, the weapon systems cannot be fully exercised.

The customer for these systems wants to know the reliability of the weapon systems in the operational context. Thus reliability is defined as:

The probability of achieving:

- the specified explosive output ("yield"),

- at the target,

- across the Stockpile To Target Sequence (STS) environments,

- throughout the weapon's lifetime.

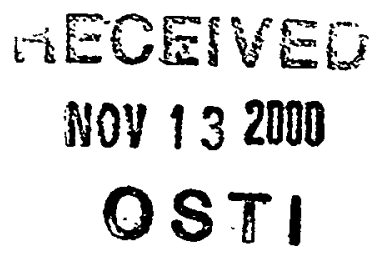

\section{Conditions:}

- The specified inputs and conditions at the interfaces are present.

- The normal environments specified by the STS have not been exceeded.

- The specified lifetime has not been exceeded.

The Stockpile to Target Sequence defines the various environments to which the weapon system could be exposed during its normal lifetime and includes transportation, handling, storage, and operational use environments. The types of environments experienced range from mechanical vibration and shock, temperature, and humidity to ionizing radiation (both low dose and transient, in some cases).

There are some challenging consequences of defining reliability in this fashion. First, the definition implies that we must assume that the probability of exposure to the worst-case set of normal STS environments is equal to one. We want to provide a realistic lower bound on reliability that applies to the entire range of conditions that the weapon system can experience. It tends to be more straightforward to examine performance at the environmental extremes by testing at the subassembly level rather than at the system level. Hence data is gathered at multiple levels of assembly and combined using a reliability block diagram model that generally has failure events defined at the subassembly level. The test program must span the range of 
environmental conditions as well as the set of weapon operational capabilities. This results in a very large state space to explore, particularly when the "throughout the lifetime" constraint is added. This is shown notionally in Figure 1. Each test that is performed gives information about a very limited area of this state space. Modeling can play an important role in leveraging this test data by allowing us to extrapolate these test results to an expanded set of conditions.

\section{Figure 1: Weapon System Reliability State Space}

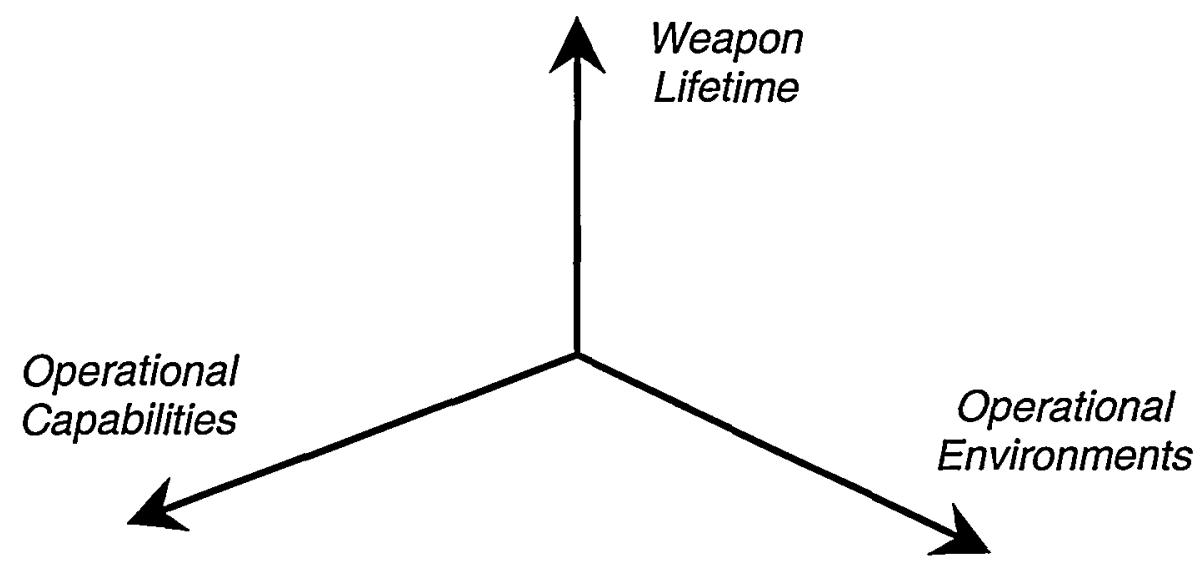

Another challenge posed by the reliability definition arises in how success is defined. The desire to relate success to "yield at the target" when such a full-up test cannot be performed means that success must always be inferred. Ideally, pass/fail specifications for testing at each level of assembly are tied to this high level definition of success, but this is difficult in practice. Here again, modeling can play an important role in developing test specification limits that are better related to overall system success.

In the context of multidecade weapon system lifetimes, degradation due to aging is a concern. There is a major program at Sandia National Laboratories examining age-related changes of materials and pieceparts to provide a predictive capability. One of the important challenges of this effort is relating any observed degradation at the material and piece-part level to weapon system performance ("yield at the target"). Note that these degradation mechanisms may be pervasive throughout a system, further complicating the analysis. These inferences are very difficult, and modeling provides an avenue by which the impact of these changes at the material and piece-part level can be evaluated in a subassembly or system context to determine if they affect reliability or simply reduce design margin.

The current reliability assessment approach uses the data from a broad set of tests that are performed at various levels of assembly, under various environmental conditions, and with various operational objectives. This test diversity allows for defect detection across the state space, but it also means that not every test is capable of detecting each of the defects present in the weapon systems. The reliability analyst must decide which test data are relevant when doing an assessment. This in turn requires an in-depth root cause analysis for each defect to help determine whether or not it could be detected in each of the test programs. Modeling provides an excellent avenue to assist with the root cause analysis process. Furthermore, modeling can be used to evaluate the generalized effect of an observed anomaly; though modeling, one can vary 
the characteristics of an anomaly, such as its severity or where it is manifested, to examine its impact in a broader sense.

In summary, the current reliability assessment approach is based upon a diverse test program in which root cause analysis plays a key role. The desire to capitalize on the information obtained through modeling (to aid in prediction of the impact of aging phenomena, to supplement the existing test data, and to better understand the data we already acquire) is the motivation behind the MBRA effort. This is particularly important since resources for testing are becoming scarcer.

\section{MBRA FRAMEWORK}

To develop the MBRA framework, reliability activities as a function of product life cycle were reviewed, and areas where the capabilities provided by MBRA could be applied were determined. This review identified the following five general MBRA processes: Design Margin Analysis, Specification Limits, Lifetime Prediction, Anomaly Investigation, and Probability Quantification. These are considered to be areas where physical response modeling can play an important role in providing cost-effective insights into reliability.

\section{Design Margin Analysis}

The Design Margin Analysis process has perhaps the lowest technical risk of any of the processes with potentially a large payoff. It can be used at almost any stage of the product life cycle when there is a desire to either quantify the range of possible performance margin of a system or subsystem or improve the performance margin of a system or subsystem. In the MBRA process, physical response modeling (in conjunction with validation testing) is used to explore the performance state space of a system. Design Margin Analysis serves as a foundation for each of the other MBRA processes.

\section{Specification Limits}

One of the variants of the Design Margin Analysis process is that of setting realistic test specification limits. This is an activity that could result in significant cost savings - the cost of overly conservative specification limits can be very high due to excess scrap and rework, unneeded inspection and screening, and selection of more expensive parts. Specifications that are not tight enough can lead to unexpected failure; even worse, these failures may not be easily detected in a test program. A long-term goal is to extend the capabilities of MBRA such that it is possible to optimize the interface specifications from a system-level perspective rather than by using a component-by-component approach.

\section{Lifetime Prediction}

Lifetime is a key decision-making and resource allocation metric for weapon systems, and improved means of predicting the impact of age-related changes are critical. The MBRA approach will be hierarchical, building a bridge between the material models and the piece-part models in order to examine the effects of aging in a subassembly- or system-level context. This 
also provides a framework to evaluate pervasive aging throughout the system (e.g., if multiple pieceparts are changing with time).

\section{Anomaly Investigation}

Physical response modeling is a natural choice for investigation of anomalies, and a variety of tools have already been used for this application. Modeling is a cost-effective way to perform root cause analysis, to examine the effect of an anomaly in a general sense, and to identify fixes. Modeling can also help to focus on what tests need to be performed and how to perform these tests.

\section{Probability Quantification}

The Probability Quantification process is the culminating step of MBRA, but it also carries with it the highest technical risk. To use physical response modeling results directly to make reliability statements, it is essential to minimize uncertainty in the results and to ensure that all salient factors are included. The results must be highly credible when major allocation of resources may be at stake. It is anticipated that initially the MBRA process for probability quantification will be used sparingly or in conjunction with other methods until its credibility is validated.

Occasionally there are anomalies for which the relationship between the observed anomalous behavior and its impact on system performance cannot be determined by testing, either because the needed test conditions are prohibitively expense or technically unachievable. In these cases, modeling could conceivably be used to make direct probabilistic statements if the phenomenon could indeed be modeled.

\section{MBRA APPLICATION}

There are many tools and approaches currently available to do physical response modeling of systems. The initial focus for MBRA has been the simulation of electrical systems using a variant of the SPICE (Simulation Program with Integrated Circuit Emphasis) code. It is expected that examination of other modeling domains (e.g., mechanical structures) will be addressed in later phases of this project. To the degree that non-electrical effects (e.g., temperature, radiation, and aging) can be modeled as variations in electrical device performance, they are included in the SPICE analyses.

MBRA processes (in particular, Design Margin and Specification Limit Analysis) are currently being used to examine an electromechanical device used in a weapon system. Results to date will be described below, along with the details of the approach.

\section{Focus of the Analysis}

The device being examined is a Trajectory Sensing Signal Generator (TSSG). It is a member of a general class of components designed by Sandia National Laboratories known as Environmental Sensing Devices (ESDs). These are very specialized devices whose purpose is to 
detect an actual use environment and to then cause contacts to be closed, allowing for weapon operation. In the absence of the actual use environment, these devices "reject" other environments and the contacts remain open. ESDs help to ensure that the weapon system does not operate except when intended.

The TSSG's function is to detect an acceleration of a certain level and duration, upon which a relay is closed. This relay then provides an electrical path for two coded pulse trains that are amplified by the TSSG before being sent to an electromechanical rotary switch that causes contacts to be closed upon receipt of the proper pulse trains. The TSSG senses acceleration using a rolamite (a mechanism consisting of rollers and a flexible band), and the duration of the acceleration is measured using a simple resistor-capacitor charging circuit as a timer. The three general functions of the TSSG are thus measuring acceleration, timing, and amplification.

\section{Modeling Issues}

A model of the TSSG was built using a commercially available version of the SPICE electrical simulation software. The model was then converted to run using Sandia's ChileSPICE. The primary motivation for using ChileSPICE is that it offers the capability of running in a multiprocessor environment; this significantly shortens execution time when doing variability analyses that may require hundreds of simulation code runs.

Some of the pieceparts in the TSSG are mechanical parts and are thus modeled in a simplistic fashion; for example, the rolamites are modeled as simple switches. The electrical pieceparts, however, are modeled in a relatively detailed manner using typical SPICE constructs to describe their electrical behavior. The electrical models for these devices also include temperature dependence and tolerance information.

The output of the SPICE transient analysis tool is a set of waveforms for each of the circuit nodes describing behavior (current and voltage) as a function of time. Since generally a single attribute of the waveform (maximum value, rise time, etc.) is desired to characterize the response, customized post-processing software is needed to extract the attribute of interest from the waveform data.

\section{Objectives of the Task}

For the prototype TSSG application, the key objectives were to exercise the Design Margin Analysis and Specification Limit MBRA processes to identify their benefits, challenges, and caveats.

The TSSG has been fielded for almost twenty years, so there are extensive test data available including $100 \%$ production acceptance tests, sample production acceptance tests at environmental extremes, and sample testing done as part of the on-going surveillance program at Sandia. The TSSG modeling approach was to emulate to the degree possible the prior test conditions (including inputs, loads, environments, monitoring points, and specific measurements) such that the model results could be reasonably compared to the actual test data. 
The production testing is detailed in a document called a Product Specification (PS), and this served as a guide for the modeling activity.

\section{$\underline{\text { Results to Date }}$}

The measurements relating to TSSG amplifier operation were examined first, since it was felt that the amplifier model was the most mature and had the best fidelity. The PS called out four different measurements to verify correct amplifier operation. These are shown notionally in Figure 2 .

Figure 2: TSSG Model Outputs (Amplifier Operation)

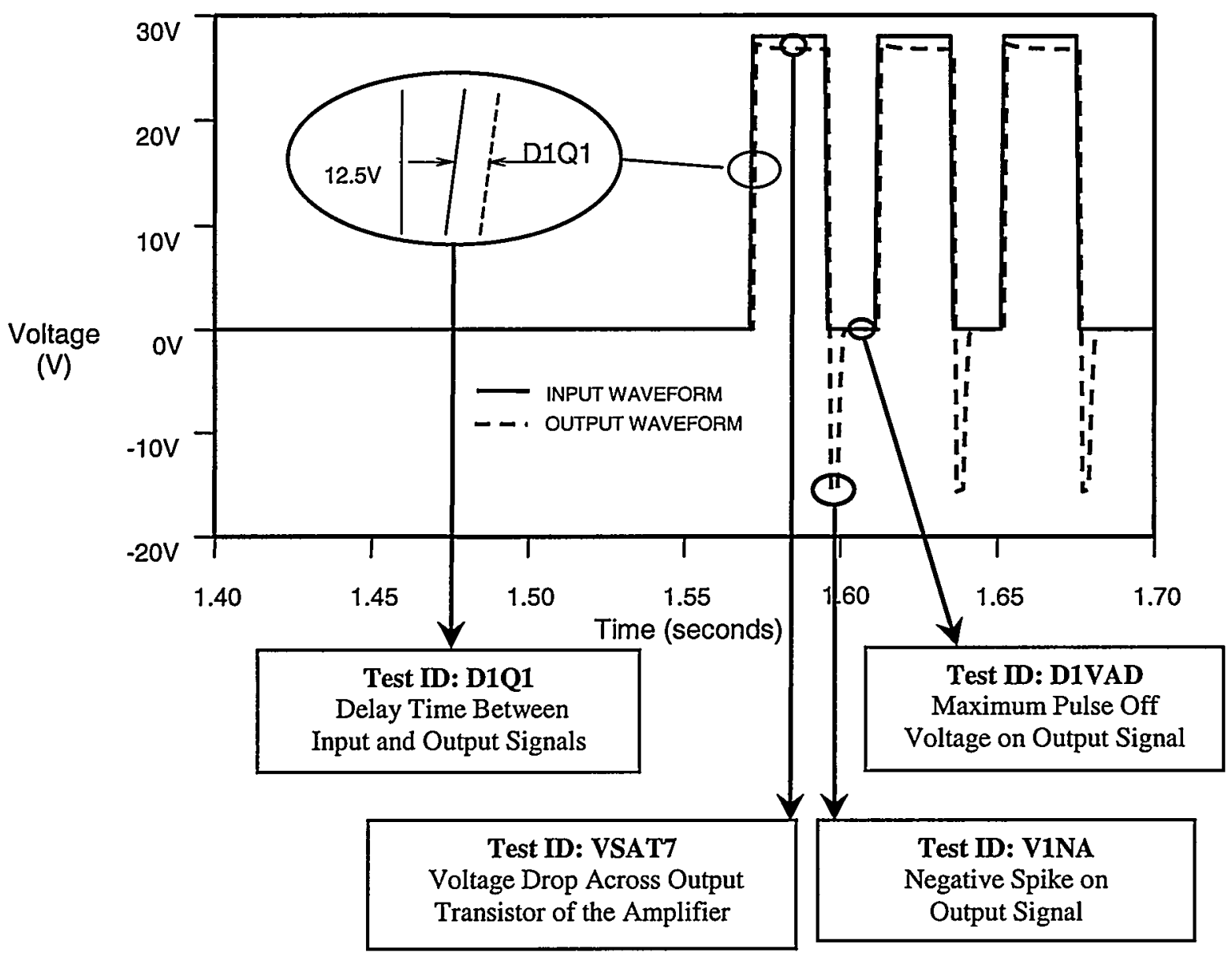

Because the PS called out tests at three different temperatures $\left(-55^{\circ} \mathrm{C}, 25^{\circ} \mathrm{C}\right.$, and $\left.75^{\circ} \mathrm{C}\right)$, the temperature-dependent model was also run under these conditions. In addition, the tolerance information was used to develop distributions for the passive devices (resistors and capacitors). The passive device values (resistance and capacitance) were assumed to be uniformly distributed across the specified tolerance band. The actual distribution is unknown but could differ appreciably from the usual assumption of a Gaussian distribution; hence it was felt that a uniform distribution provided a more conservative estimate. For the semiconductor devices, high, low, and nominal models were used to represent the tolerance variability. A Sandia- 
developed sensitivity and uncertainty analysis tool, SUNS@, generated the variability analysis test cases. Latin Hypercube sampling (a structured variant of Monte Carlo sampling) was used to ensure complete coverage of the parameter distributions.

A comparison of the production data and modeling results is shown in Figure 3 for the D1Q1 measurements. The limits on the graph are the specifications called out in the PS for D1Q1. The whiskers shown in the graph encompass the middle $80 \%$ of the data distribution, with data values outside of this range shown as individual points. The boxes encompass the middle $50 \%$ of the data range, and the line in the box is the median value of the data. Note that one test data point taken at $75^{\circ} \mathrm{C}$ failed to meet the specification; it is shown in the graph below outside of the PS limits.

Figure 3: Comparison of Model and Test Results

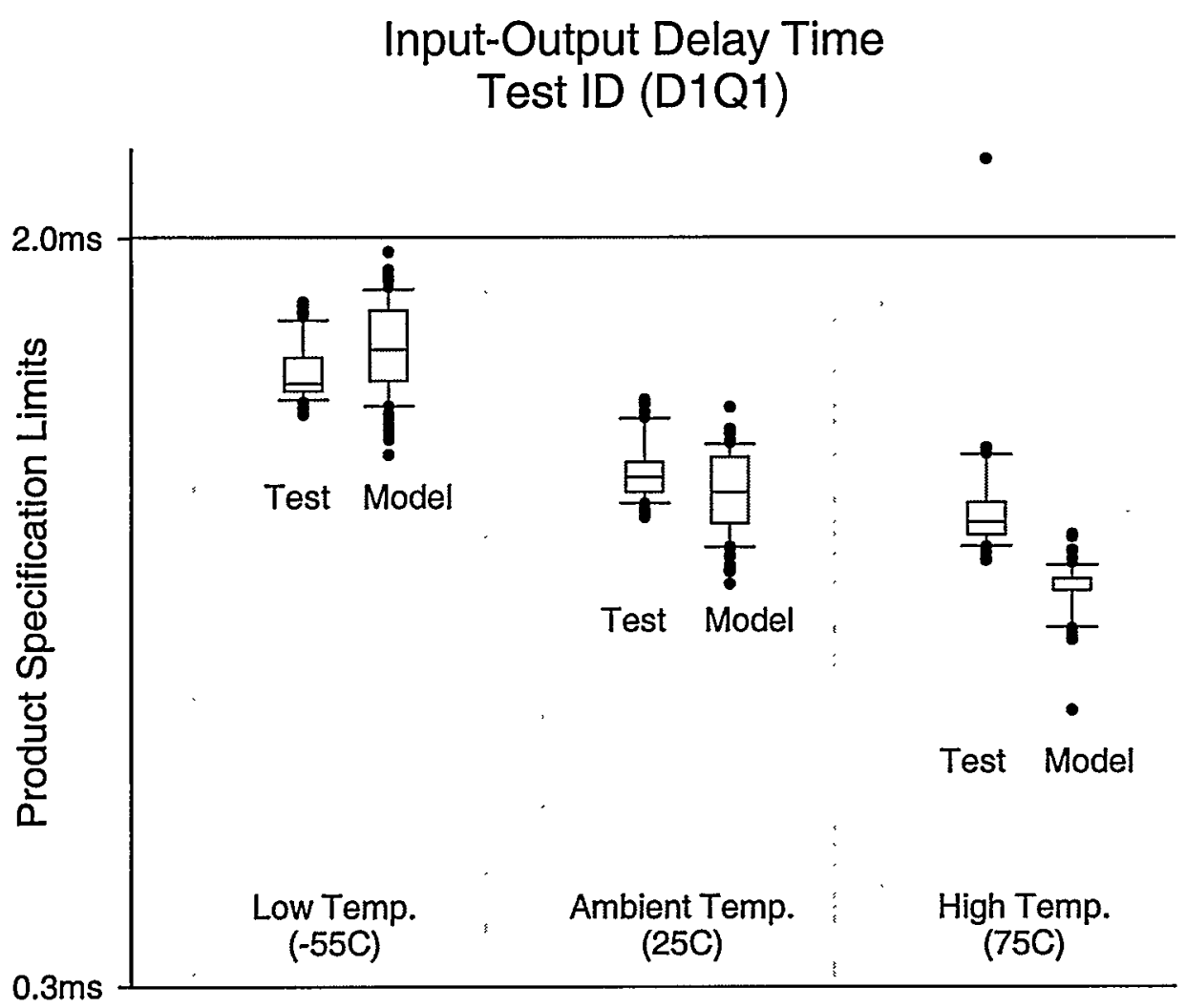

Two different testers have been used over the life of the TSSG. For some of the measurements (in particular, VSAT7, the voltage drop across the output transistor), the distributions of the data taken by the two different testers have different center values and widths. The cause of this discrepancy is unknown. It could be due to differences between the testers, different test equipment operators, or actual changes in the product over time. This highlights one of the difficulties of comparing model and test results: models allow for "perfect" instrumentation, while testers always introduce some effect which often is not quantified or well-understood. 


\section{Implications and Observations}

Analysis of the data is still underway. The goals are:

- identify design margins under different conditions

- identify key parameters that impact TSSG performance

- determine if specifications need to be modified to better map to "yield at the target"

- identify potential reliability issues

- identify possible enhancements to the on-going surveillance activities

An example of one aspect of the data analysis is shown in Figure 4. The SUNS tool allows one to examine correlations between input parameters and each output. This provides the capability of identifying important contributors to the outputs of interest, as well as the nature of the contribution (i.e., if the parameters are positively correlated or negatively correlated with the outputs). For the example in Figure 4, the capacitor C102 (CBH1_C102) has a correlation coefficient of approximately 0.9 , indicating that it is a strong contributor to the variability of the $\mathrm{D} 1 \mathrm{Q} 1$ measurement at $25^{\circ} \mathrm{C}$. As the capacitance value of C102 increases, the delay time D1Q1 increases. Similar analyses can be done for each of the measurements. In addition, analyses can be performed to determine the primary contributors to the temperature dependence that is evident in the graph in Figure 3.

\section{Figure 4: Correlation Results for Output D1Q1 $\left(25^{\circ} \mathrm{C}\right)$}

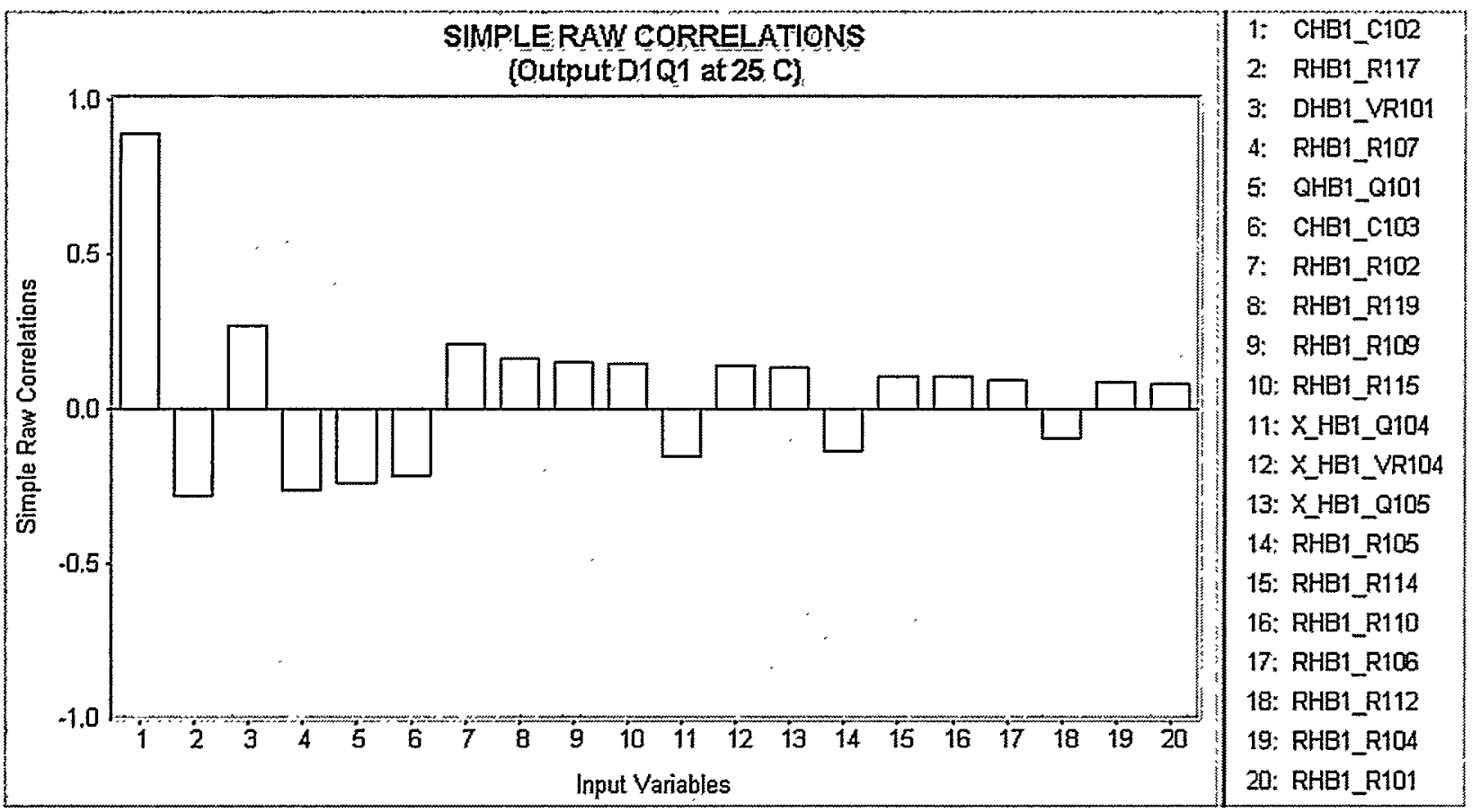

One clear outcome of the work to date is that modeling and testing have a strong synergistic relationship. The various test activities provide data suitable for model validation for various 
conditions of interest. On the other hand, validated models can help to leverage the test data through (appropriate) extrapolation to other conditions.

\section{NEXT STEPS}

An important step currently being pursued is that of validation of the model results using the data already acquired. Note that there are some concerns based on the bimodal distribution of some of the test data (as described earlier) and the lack of knowledge regarding the effect of the tester on the measurements. Once the model is validated, the MBRA Design Margin and Specification Limit Analyses to identify key parameters and examine specifications can proceed for the TSSG. Longer-term goals include analysis of the TSSG in conjunction with its interfaces to examine synergistic effects. It is also planned to eventually incorporate other physical response modeling domains (e.g., mechanical or thermal).

\section{ACKNOWLEDGMENTS}

We would like to thank others at Sandia National Laboratories who participated in this effort. In particular, Michele Caldwell, Seung Choi, Elmer Collins, Chuck Harjes, Ken Marx, David Smith, and John Tenney provided invaluable support.

\section{BIOGRAPHIES}

Rene L. Bierbaum

Sandia National Laboratories

P.O. Box 969 , MS9202

Livermore, CA 94551-0969

925-294-3802

rlbierb@sandia.gov

Ms. Bierbaum received a B.S. in Electrical Engineering from the University of Nebraska in 1983 and an M.S. in Electrical Engineering from Stanford University in 1984. She is a Principal Member of the Technical Staff at Sandia National Laboratories in Livermore, CA. She is currently affiliated with the Reliability and Electrical Systems Department at Sandia, working as a reliability analyst for the nuclear weapons program.

Thomas D. Brown

Sandia National Laboratories

P.O. Box 5800, MS0405

Albuquerque, NM 87185

505-844-6134

tdbrown@sandia.gov

Mr. Brown received a B.S. in Chemical Engineering from the University of New Mexico in 1984 and an M.S. in Mechanical Engineering from the University of California at Berkeley in 1987. He is a Principal Member of the Technical Staff at Sandia National Laboratories in 
Albuquerque, NM. He works as a surety analyst in the Weapon Surety Engineering Department at Sandia.

Thomas J. Kerschen

Sandia National Laboratories

P.O. Box 5800, MS0830

Albuquerque, NM 87185

505-844-3706

tjkersc@sandia.gov

Mr. Kerschen received a B.A. in Mathematics from the New Mexico State University in 1975 and an M.S. in Mathematics from the University of Washington in 1977. He is a Principal Member of Technical Staff at Sandia National Laboratories in Albuquerque, NM. He is currently in the Reliability Assessment Department at Sandia working as a reliability analyst for the nuclear weapons program. 